Direct evidence for the participation of band-tails and excited-state tunnelling in the luminescence of irradiated feldspars

This article has been downloaded from IOPscience. Please scroll down to see the full text article.

2009 J. Phys.: Condens. Matter 21485505

(http://iopscience.iop.org/0953-8984/21/48/485505)

View the table of contents for this issue, or go to the journal homepage for more

Download details:

IP Address: 131.180.130.109

The article was downloaded on 08/08/2011 at 09:52

Please note that terms and conditions apply. 


\title{
Direct evidence for the participation of band-tails and excited-state tunnelling in the luminescence of irradiated feldspars
}

\author{
N R J Poolton ${ }^{1,2}$, R H Kars ${ }^{1,3}$, J Wallinga ${ }^{1,3}$ and A J J Bos ${ }^{1}$ \\ ${ }^{1}$ Faculty of Applied Sciences, Delft University of Technology, Mekelweg 15, 2629 JB Delft, \\ The Netherlands \\ ${ }^{2}$ Institute of Mathematics and Physics, Aberystwyth University, Aberystwyth SY23 3BZ, UK \\ ${ }^{3}$ Faculty of Applied Sciences, The Netherlands Centre for Luminescence Dating, \\ Delft University of Technology, Mekelweg 15, 2629 JB Delft, The Netherlands
}

Received 26 August 2009, in final form 20 October 2009

Published 11 November 2009

Online at stacks.iop.org/JPhysCM/21/485505

\begin{abstract}
The significance and extent of band-tail states in the luminescence and dosimetry properties of natural aluminosilicates (feldspars) is investigated by means of studies using low temperature $(10 \mathrm{~K})$ irradiation and optically stimulated luminescence (OSL) stimulation spectroscopy, and thermoluminescence (TL) in the range 10-200 K, made in comparison with high temperature $(300 \mathrm{~K})$ irradiation and photo-transferred OSL and TL investigations undertaken at low temperature. These measurements allow mappings of the band-tails to be made; they are found to be $\sim 0.4 \mathrm{eV}$ in extent in the typical materials studied. Furthermore, by populating charge trapping centres at high temperature $(300 \mathrm{~K})$ and monitoring the OSL stimulation spectra at temperatures in the range $10-300 \mathrm{~K}$, clear evidence is presented for the presence of both thermally activated and non-thermally activated OSL processes; it is argued that the former result from thermally activated hopping through the band-tail states, whilst the latter are due to tunnelling processes, either from the excited state of the OSL centres or through the tail states. The spectral measurements are supported by analysis of the temporal dependence of the OSL signals, which correspond to either tunnelling or general order kinetic decay processes.
\end{abstract}

\section{Introduction}

Feldspars are wide band-gap aluminosilicates $\left(E_{\mathrm{g}} \sim 7.7 \mathrm{eV}\right.$; Malins et al 2004) which occur ubiquitously in nature. Owing to the presence of energetically deep-lying defects within the band-gaps that have the capability to trap and store charge over time, they have long been considered as potential materials for retrospective dosimetry applications such as geological optical dating (e.g. Aitken 1998). However, in most feldspars, quantum-mechanical tunnelling between electron and hole trapping centres leads to the loss of what would normally be regarded as thermally time-stable charge (Wintle 1973, Huntley and Lamothe 2001) and this greatly complicates reliable dosimetry measurements: in such applications, the use of quartz (if available, and which has a similar band-gap energy $E_{\mathrm{g}} \sim 8.7 \mathrm{eV}$; Itoh et al 1989) is nearly always the preferred mineral of choice due to the substantially improved time-stability of the trapped charge. On the plus side, feldspars do tend to have much higher radiation saturation thresholds than quartz (offering the possibilities of dating older materials), and in certain applications-such as potential in situ dating of Martian sediments (e.g. McKeever et al 2003), there may be no quartz present and so feldspars may represent the only suitable natural dosimeters available. In order to progress the optical dating of feldspars, there is therefore a clear need to understand why tunnelling charge loss occurs in these materials, or at the very least provide effective diagnosis of the fading (charge loss) problem.

Unlike crystalline quartz, which has a rigid lattice framework and well-defined electronic energy structure, the feldspars have much more open lattice networks, resulting in a significantly relaxed range of bonding angles (Megaw 1974). Also unlike quartz, one of the main optically active defects that is ubiquitously present in the minerals - and that can be used for optical dating - is known to have at least one excited state that can be probed with infra-red light (e.g. Hütt et al 1988, Clark and Sanderson 1994, Barnett and Bailiff 1997, Poolton et al 1995a, Baril and Huntley 2003). If promoted to these 

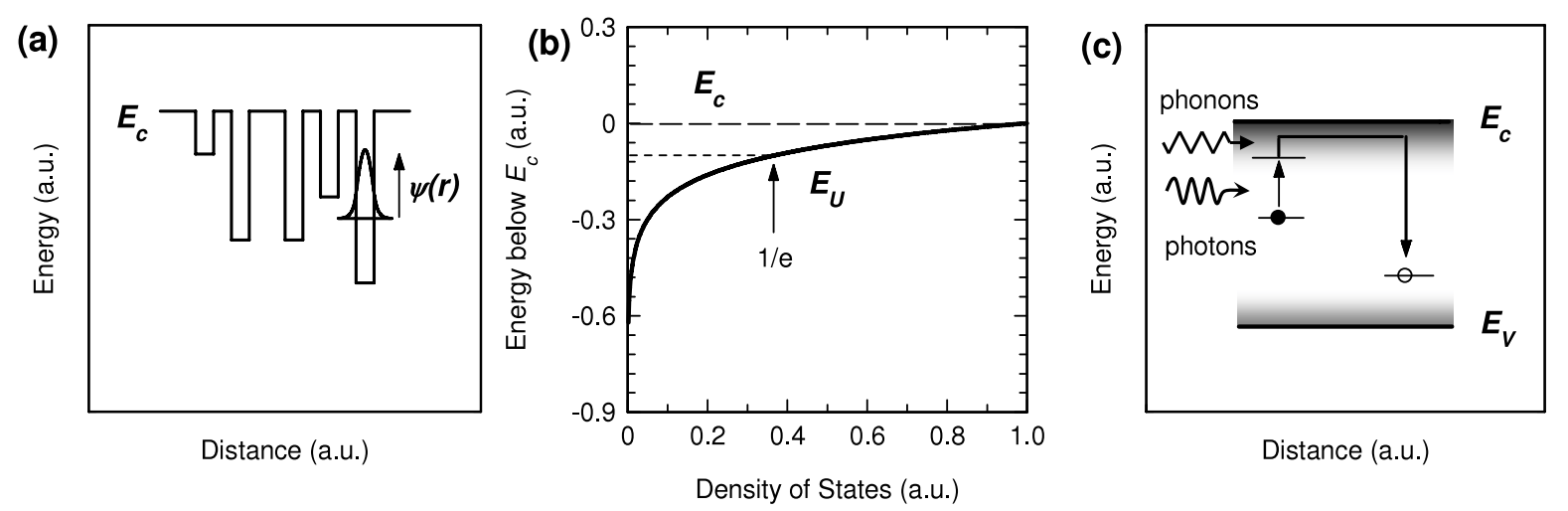

Figure 1. Basic concepts of the feldspar traps and band-tails discussed in the paper. (a) Fluctuations in bonding angle throughout the crystal structure generate localized shallow states below the high mobility conduction band edge $E_{\mathrm{c}}$ that can trap electrons at low temperature: as the wavefunction, $\psi(r)$, of these electrons extend beyond the well boundaries, this allows tunnelling between the states without accessing the high mobility edge. (b) The states form a continuum, whose density is usually exponentially variable with energy below $E_{\mathrm{c}}$ : the width of this Urbach tail is defined as $E_{\mathrm{U}}$. (c) The additional presence of a specific defect trap in feldspars that possesses an excited state below $E_{\mathrm{c}}$ means that optically stimulated luminescence can be probed photo-thermally via the state; if the excited state is embedded in the band-tails, then recombination can be either thermally activated (hopping amongst the band-tails) or non-thermal (tunnelling either between the band-tails, or directly to the recombination centre).

excited states, trapped electrons can proceed to recombine at hole trapping centres, causing the emission of optically stimulated luminescence (OSL or, in the infra-red range, IRSL) on which the dating method relies; essentially, the older the material, the more trapped charge, and thus the brighter the signal. Previously, we have argued that, theoretically, the bonding angle variations, and the presence of the excited defect states should combine to explain many observed complex features of the photo-thermal dependence of charge mobility and luminescence in feldspars (Poolton et al 2002). It was argued that different bonding angles would result in localized states within the band-gap, and a continuum of angle variations would result in a continuum of ('band-tail') states, extending both below the high mobility edge of the conduction band, and above the valence band. Furthermore, the excited state of the optically active defect would normally be expected to reside within this band-tail, allowing transport out of the centre, without actually gaining enough energy to access the high mobility conduction band edge. It was proposed that this could have ramifications for dosimetry, including the increased possibility of direct tunnelling to nearby centres from the excited state. The concepts of the band-tails and the photothermal transitions are summarized in figure 1.

Despite the theoretical models that predict the presence of band-tails and the possibility for excited-state tunnelling processes, there has hitherto been little direct experimentally derived evidence to prove their existence, and thus their importance in the luminescence dosimetry of feldspars has remained elusive. The main reason for this is that, in order to probe and characterize the effects decisively, radiation dosimetry measurements would need to be undertaken in the especially crucial temperature range $10-70 \mathrm{~K}$, rather than from 300 to $600 \mathrm{~K}$ that is mostly commonly referred to in the literature (and summarized by Bøtter-Jensen et al 2003) or in the more easily accessible 140-300 K range (Bailiff and Barnett 1994, Rieser et al 1997); in fact, we are aware of only a single instance of thermoluminescence having previously been undertaken from 10 to $100 \mathrm{~K}$ in feldspars by Visocekas et al (1998). The purpose of this paper is therefore to undertake a survey of low temperature OSL and TL measurements in representative feldspars for which the results can be definitively linked with those predicted theoretically concerning the involvement of band-tail states and excitedstate tunnelling process.

\section{Experimental details}

The experimental arrangement used is such as to allow x-ray excitation, optical stimulation and luminescence detection at all temperatures from 10 to $300 \mathrm{~K}$. The kit assembled to do this is essentially the same as that described by Poolton et al (2009) and, briefly, is comprised of a variable temperature closedcycle cryostat (Advanced Research Systems, DE202NB: 10$350 \mathrm{~K})$ with multiple $\mathrm{x}$-ray and optical ports. The x-ray source is a $2 \mathrm{~kW}$ Philips PW2273/20 x-ray tube with copper target, producing both bremsstrahlung radiation (limited to a maximum of $30 \mathrm{keV}$ in this experiment) and $\mathrm{Cu} \mathrm{K} \alpha$ at $8 \mathrm{keV}$. Between the source and the cryostat x-ray window (aluminized mylar, $100 \mu \mathrm{m}$ thick) is an air gap, into which a computercontrolled X-ray shutter is installed (Uniblitz XRS25); this has a full-aperture switching speed of $10 \mathrm{~ms}$ and, with platinum/iridium blades, effectively blocks x-rays with energy below $30 \mathrm{keV}$. Just after the divergent $\mathrm{x}$-ray source, and just prior to the cryostat $\mathrm{x}$-ray window are steel apertures that act as collimators and restrict the x-ray excitation area of the sample to about $2 \mathrm{~mm}^{2}$. Based on the manufacturer's specifications, the total $\mathrm{x}$-ray power received at the sample would be approximately $70 \mu \mathrm{W} \mathrm{cm}{ }^{-2}$.

Wavelength-resolved OSL stimulation spectra were achieved by means of a $19 \mathrm{~cm}$ Jobin-Yvon Triax scanning spectrometer coupled to a white light broadband source $(20 \mathrm{~W}$ tungsten halogen lamp); monochromatic excitation densities at the sample were limited to between 60 and $90 \mu \mathrm{W} \mathrm{cm}{ }^{-2}$, 
(a)
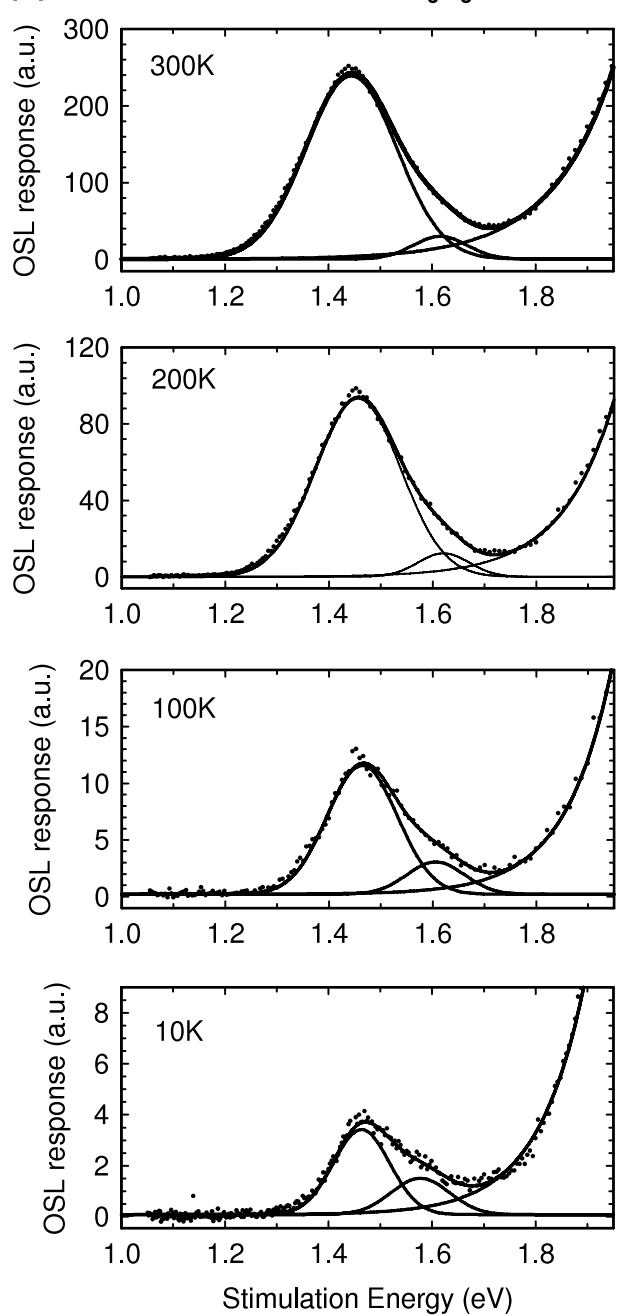

(b) $\quad \mathrm{R28:} \mathrm{KAISi}_{3} \mathrm{O}_{8}$
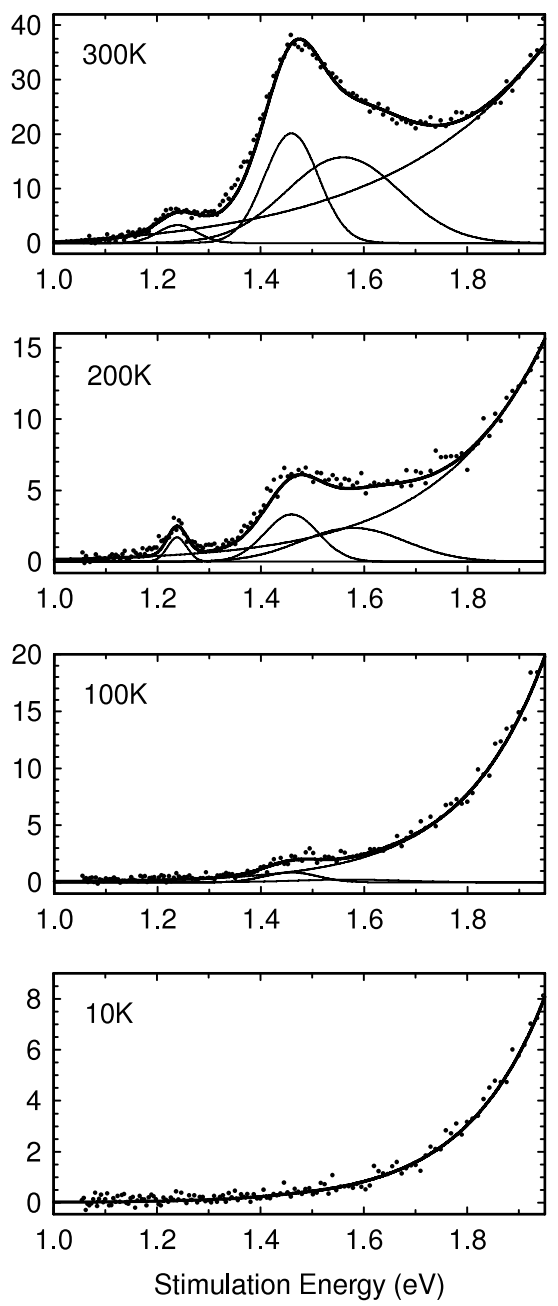

Figure 2. OSL stimulation spectra in $\mathrm{K}$ and $\mathrm{Na}$ feldspars, for materials irradiated at $300 \mathrm{~K}$ but measured in the temperature range $10-300 \mathrm{~K}$. Solid lines show the fitted curves to the data, which comprise both resonant and non-resonant (continuum) signals.

depending on wavelength in the range $635-1200 \mathrm{~nm}$ (1$1.95 \mathrm{eV})$; the stimulation spectra have been corrected for this variation in power density. Such low power densities result in minimal fractional charge loss during each OSL excitation scan. For OSL time-decay measurements, the monochromator slits can be widened to realize 2-4 times the flux. The inclusion of a $610 \mathrm{~nm}$ long pass filter prevents stimulation with second order (higher energy) photons, up to $1200 \mathrm{~nm}$ (primary wavelength).

OSL is detected via a blue/green sensitive photomultiplier with bialkali photocathode response operating in photoncounting mode, and filtered with $6 \mathrm{~mm}$ of Schott UG11 colour glass, which provides an optical detection window between 300 and $380 \mathrm{~nm}$.

The individual samples used were cleaved museum specimens of single crystal feldspars (typical dimensions $2 \mathrm{~mm} \times 2 \mathrm{~mm} \times 1 \mathrm{~mm}$ ), and are chosen to be both representative of their genre, and demonstrate the range of physical effects discussed. They are; $\mathrm{NaAlSi}_{3} \mathrm{O}_{8}$ (sodium feldspar, albite; reference $\mathrm{R} 27$ ), and $\mathrm{KAlSi}_{3} \mathrm{O}_{8}$ (potassium feldspar, orthoclase; reference R28). Previous complimentary high temperature measurements have been undertaken on these particular samples, and further mineralogical information can be found in Poolton et al (1995a, 1995b): it should be noted that R12 in these references is equivalent to R27 used here.

\section{Results and discussion}

\subsection{Probing excited-state photo-thermal and tunnelling processes}

Following x-irradiation at room temperature and a subsequent 20 min delay to allow any phosphorescence to decay, the optical stimulation spectra for the OSL in both R27 and R28 (15 and $50 \mathrm{~min} \mathrm{x}$-ray exposure, respectively) are presented in figure 2, as a function of temperature in the range 10-300 K, and stimulation range $1-1.95 \mathrm{eV}$. At $300 \mathrm{~K}$, the spectra are comprised of a number of resonances, superimposed on a rising continuum: the latter is considered explicitly in the following section; here we are concerned only with the resonances themselves.

In the case of $\mathrm{NaAlSi}_{3} \mathrm{O}_{8}$ (sample R27), the spectrum is fitted to two overlapping Gaussian transitions, the dominant 
(a)

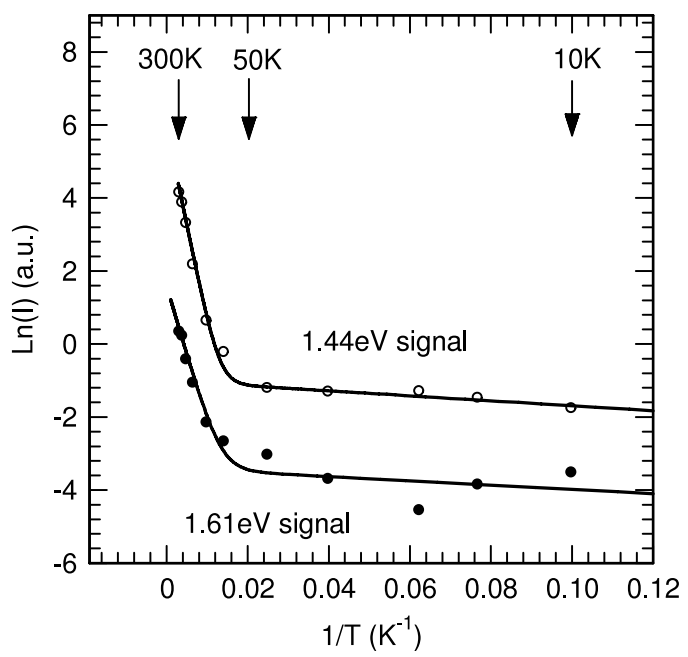

(b) R28: $\mathrm{KAISi}_{3} \mathrm{O}_{8}$

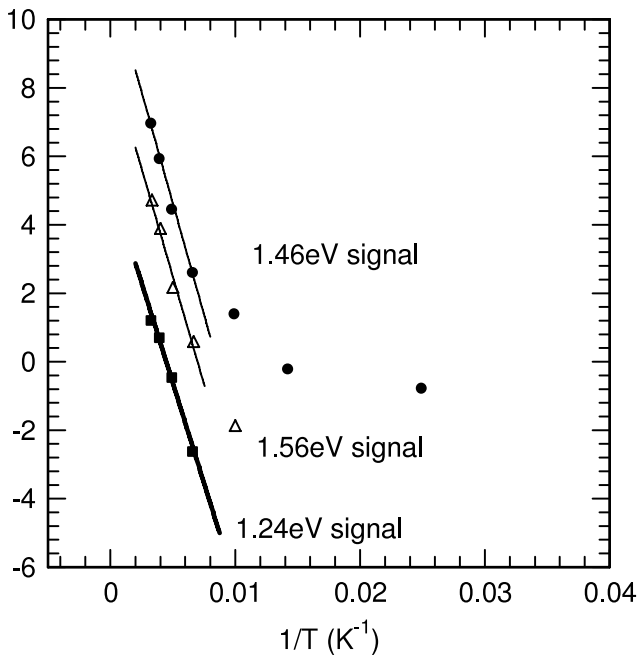

Figure 3. Arrhenius plots of the integrated intensities of the resonant transitions in (a) Na and (b) K feldspar, clearly showing the presence of a thermally activated process dominant above $\sim 80 \mathrm{~K}$, and a non-thermal process dominant at temperatures below $\sim 80 \mathrm{~K}$.

peaking at $1.444 \mathrm{eV}(\mathrm{FWHM}, 0.21 \mathrm{eV})$ with a secondary minor peak at $1.61 \mathrm{eV}\left(\mathrm{FWHM}, 0.12 \mathrm{eV}\right.$ ). For the $\mathrm{KAlSi}_{3} \mathrm{O}_{8}$ (sample R28), three overlapping Gaussian transitions were observed, peaking at $1.46 \mathrm{eV}$ (FWHM, $0.12 \mathrm{eV}$ ), $1.56 \mathrm{eV}$ (FWHM, $0.26 \mathrm{eV}$ ) and a third, weaker resonance appearing at $1.24 \mathrm{eV}$ (FWHM, $0.09 \mathrm{eV}$ ).

These OSL stimulation features are typical of those commonly found in both $\mathrm{Na}$ and $\mathrm{K}$ feldspars, with almost identical resonances having been reported in a number of different cases. For example, Baril and Huntley (2003) found transitions at 1.44 and $1.58 \mathrm{eV}$ in a number of $\mathrm{Na}$ feldspars (more precise figures are given for the individual samples), and at 1.44 and $1.54 \mathrm{eV}$ in K-rich materials - together with a third weaker transition occurring at $\sim 1.35 \mathrm{eV}$. Barnett and Bailiff (1997) found transitions at 1.455 and $1.595 \mathrm{eV}$ in Na feldspars, and at typically 1.45 and $1.54 \mathrm{eV}$ in a number of $\mathrm{K}$ feldspars (again, more precise figures are given for individual samples). The linewidths of these various transitions are also very similar to those measured in the present work. These aspects combine to indicate that samples R27 and R28 being studied in this work are truly representative of $\mathrm{Na}$ and $\mathrm{K}$ feldspars, respectively.

On cooling the samples below $300 \mathrm{~K}$, there are small systematic shifts in the energy positions and linewidths of some of the peaks, the latter being quite obvious in figure 2(b) from the resolved transition in $\mathrm{R} 28$ at $1.24 \mathrm{eV}$, between 300 and $200 \mathrm{~K}$. The linewidth and energy position shifts however are regarded as being second order effects, and not explicitly considered further in this work. The most relevant consideration regards the integrated intensities of the identified spectral components, and the temperature variable Arrhenius plots for these are presented in figure 3 for both R27 and R28. Note that OSL involves both excitation and recombination of charge, and both processes may contribute to any thermal dependence of the overall OSL signals. However, the efficiency of the excitation part of the cycle is the main concern of the present work, not the efficiency of luminescence within the recombination centre; the latter can readily be examined by monitoring the intensity of the x-ray generated luminescence as a function of temperature, and the data shown in figure 3 have been corrected for this efficiency change. This figure therefore monitors only the thermal dependence of the OSL excitation process and contains two sets of crucial information; between 100 and $300 \mathrm{~K}$, the transitions display thermally activated process, leading to the thermo-optically stimulated charge-carrier depopulation process indicated schematically in figure 1(c). For $\mathrm{NaAlSi}_{3} \mathrm{O}_{8}$, thermal activation energies of 0.04 and $0.03 \mathrm{eV}$ (both $\pm 0.01 \mathrm{eV}$ ) are determined for the $1.44 \mathrm{eV}$ and $1.61 \mathrm{eV}$ transitions, respectively. In the case of $\mathrm{KAlSi}_{3} \mathrm{O}_{8}$, thermal activation energies of $0.11 \mathrm{eV}, 0.11 \mathrm{eV}$ and $0.10 \mathrm{eV}$ (all $\pm 0.01 \mathrm{eV}$ ) are determined for the $1.46 \mathrm{eV}, 1.56 \mathrm{eV}$ and $1.24 \mathrm{eV}$ optical transitions respectively. Thermal activation energies in $\mathrm{K}$ feldspar of between 0.1 and $0.2 \mathrm{eV}$ have been previously reported for OSL excitation at $\sim 1.4 \mathrm{eV}$ in the temperature range 140-500 K, with some sample-to-sample variation, and dependence of the temperature window of study (e.g. Bailiff and Barnett 1994, Poolton et al 1995b, Rieser et al 1997) and so the values measured here appear typical.

The new piece of information to derive from the present work concerns what happens to these transitions below $100 \mathrm{~K}$; this is most clearly identified in R27, with the continued presence of strong resonant transitions observable even to $10 \mathrm{~K}$. These low temperature IRSL signals cannot be associated with the classical view of a photo-thermal process. Indeed, the Arrhenius plots in figure 3(a) show unequivocally that both transitions have a second non-thermal component, with $0.00 \mathrm{eV}$ activation energy. (Free fitting the two Arrhenius plots in the range $10-80 \mathrm{~K}$ yields apparent thermal activation energies of $0.0005 \mathrm{eV}$, but this is within the context of $\pm 0.005 \mathrm{eV}$ experimental error.) The resonant transitions are too weak to be observed in the $\mathrm{KAlSi}_{3} \mathrm{O}_{8}$ sample at $10 \mathrm{~K}$, but the $1.46 \mathrm{eV}$ signal is intense enough to indicate that there is also a non-thermal component active, with the integrated signal intensity below $100 \mathrm{~K}$ deviating strongly on the Arrhenius plot, from the thermally activated process in the range 100-300 K. 


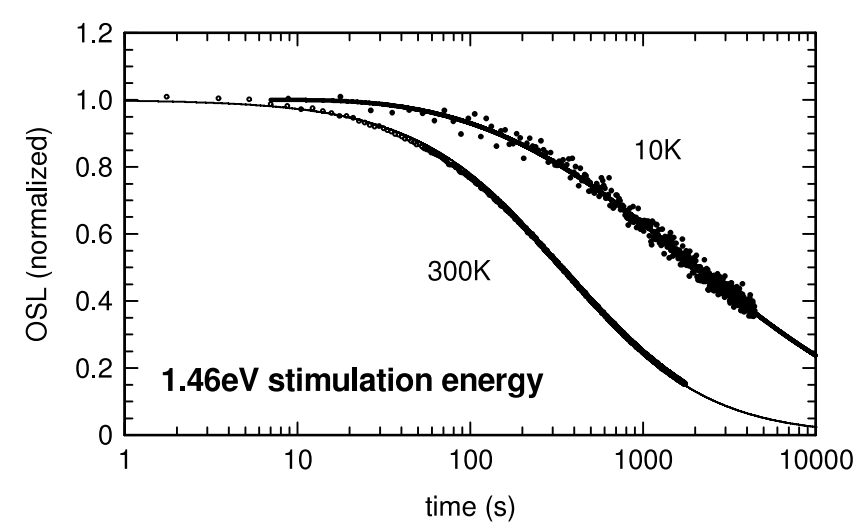

Figure 4. OSL time-decay curves for the $\mathrm{NaAlSi}_{3} \mathrm{O}_{8}$ sample; the photon energy used is $1.46 \mathrm{eV}$. In each case, irradiation has been undertaken at $300 \mathrm{~K}$, but the OSL is undertaken at either 10 or $300 \mathrm{~K}$. The time-decay functions in each case are entirely different, and are fitted (continuous lines) to general order kinetics (equation (1)) at $300 \mathrm{~K}$, or that of a tunnelling process (equation (2)) at $10 \mathrm{~K}$. System parameters are given in the text.

Two possible mechanisms will be explored that can explain the transition from thermally active to thermally inactive OSL as the sample temperature is reduced, both of which are quantum mechanically based. The least likely of these involves consideration of the inherent zero-point energy, and will be considered in section 3.2. However, we consider that the most obvious explanation of a nonthermal infra-red resonant transition in the feldspars is a direct luminescent tunnelling process from the electron trapping centre's excited state to the recombination centre. If this is the case, the dynamics of the thermally assisted and nonthermal (tunnelling) OSL would be expected to be radically different. In the case of $\mathrm{R} 27\left(\mathrm{NaAlSi}_{3} \mathrm{O}_{8}\right)$ this difference is exemplified by the OSL time-decay curves shown in figure 4; here the excitation energy is set close to the peak of the main IR resonance $(1.46 \mathrm{eV})$, but the measurement is taken at either $10 \mathrm{~K}$ (where the non-thermal photo-activated processes dominant) or $300 \mathrm{~K}$ (where the photo-thermal processes dominate). At $300 \mathrm{~K}$, the time-dependent OSL decay follows the general order kinetics equation:

$$
\operatorname{OSL}(t)=\frac{\operatorname{OSL}(0)}{(1+\alpha t)^{\beta}}
$$

where OSL(0) is the OSL intensity on activation of the stimulation light at $t=0, \alpha$ is a parameter that includes information on the initial charge population, capture cross section and intensity of the excitation light, fitted here to 0.0024 , whilst $\beta$ is a parameter that contains information regarding the order of the kinetics, and generally lies between 1 and 2: here it is fitted to 1.16 .

For measurements taken at $10 \mathrm{~K}$, there are no parameters in which the OSL time-decay could be fitted to this equation, and we conclude the OSL does not follow the form of equation (1). However, if this non-thermal OSL emission is indeed a tunnelling process, the function $\operatorname{OSL}(t)$ should be indicative of the remnant trapped charge population; this situation has been explored by both Huntley (2006) and Kars et al (2008), for the case of ground-state tunnelling processes, but the processes would be equally applicable for tunnelling from excited states, where the stimulation flux that allows excitation of charge from the ground to the excited state is kept constant. As such, the OSL would be expected to follow the form:

$$
\operatorname{OSL}(t)=\operatorname{OSL}(0) \exp \left\{-\gamma[\ln (\eta t)]^{3}\right\} .
$$

Here $\gamma$ and $\eta$ are system constants and, for a process involving the ground state, relate to the density of defects and the attempt-to-escape frequency factor of the trapped charge respectively. Tunnelling from an optically excited state of the defect would be expected to follow a similar form, but where the values of the constants change. These changes reflect the rate of excitation from the ground-to-excited state, and the time spent in the excited state (both contributing to $\eta$ ) and the difference in apparent density of defects due to a different spatial volume occupied by an electron $(\gamma)$. The data in figure 4 taken at $10 \mathrm{~K}$ indicates that this function provides a satisfactory fit to the available data, with the fitting constants being $\gamma=0.0037$ and $\eta=0.148 \mathrm{~s}^{-1}$. For comparison with tunnelling from the ground state, $\eta$ is typically in the region of $10^{15} \mathrm{~s}^{-1}$; most of this difference can potentially be accounted for by the excitation flux (at $1.4 \mathrm{eV}$ ) of $\sim 3 \times$ $10^{14}$ photons s $\mathrm{sm}^{-1} \mathrm{~cm}^{-2}$; the relaxation time-constant from the excited state is unknown at present.

\subsection{Optically probing the band-tail states from the OSL defect ground state}

For OSL transitions from the ground state of an electron trapping centre (of depth $E_{\mathrm{d}}$ ) to the conduction band in a pristine material containing no band-tail states, the excitation energy dependence of the luminescence efficiency, $\operatorname{OSL}(E)$ will be dominated by the density of states in the conduction band and, where this is parabolic, is generally expected to follow the form:

$$
\operatorname{OSL}(E) \propto \frac{\left(E-E_{\mathrm{d}}\right)^{1 / 2}}{E^{\phi}} .
$$

Here, $\phi$ is a constant that depends on which model for trapping is used (e.g. see Bøtter-Jensen et al 2003), but the crucial point is that there should be a clear threshold for the commencement of OSL stimulation from the traps at $E_{\mathrm{d}}$. The optical trap depth $E_{\mathrm{d}}$ has never been determined accurately in feldspars. Using thermal bleaching of the OSL signals, the traps have been affirmatively associated with common timestable TL traps appearing between 570 and $700 \mathrm{~K}$ (see Hütt et al 1988, Bailiff and Poolton 1991, Duller and Wintle 1991) and these would have thermal depths of between $\sim 1.6$ and $1.8 \mathrm{eV}$ (Strickertsson 1985). Based on a configurationalcoordinate model, the optical trap depth will certainly be greater than this. By experimentally examining the rate of OSL decay as a function of stimulation energy, Baril and Huntley (2003) estimated that $E_{\mathrm{d}}>2.5 \mathrm{eV}$. Furthermore, Poolton et al (1995b) showed that $2.5 \mathrm{eV}$ is also the photon energy in which the thermal activation energy of OSL reaches a watershed, being constant above this photon energy, and increasing below it. Conversely, based partly on theoretical models, Hütt et al 

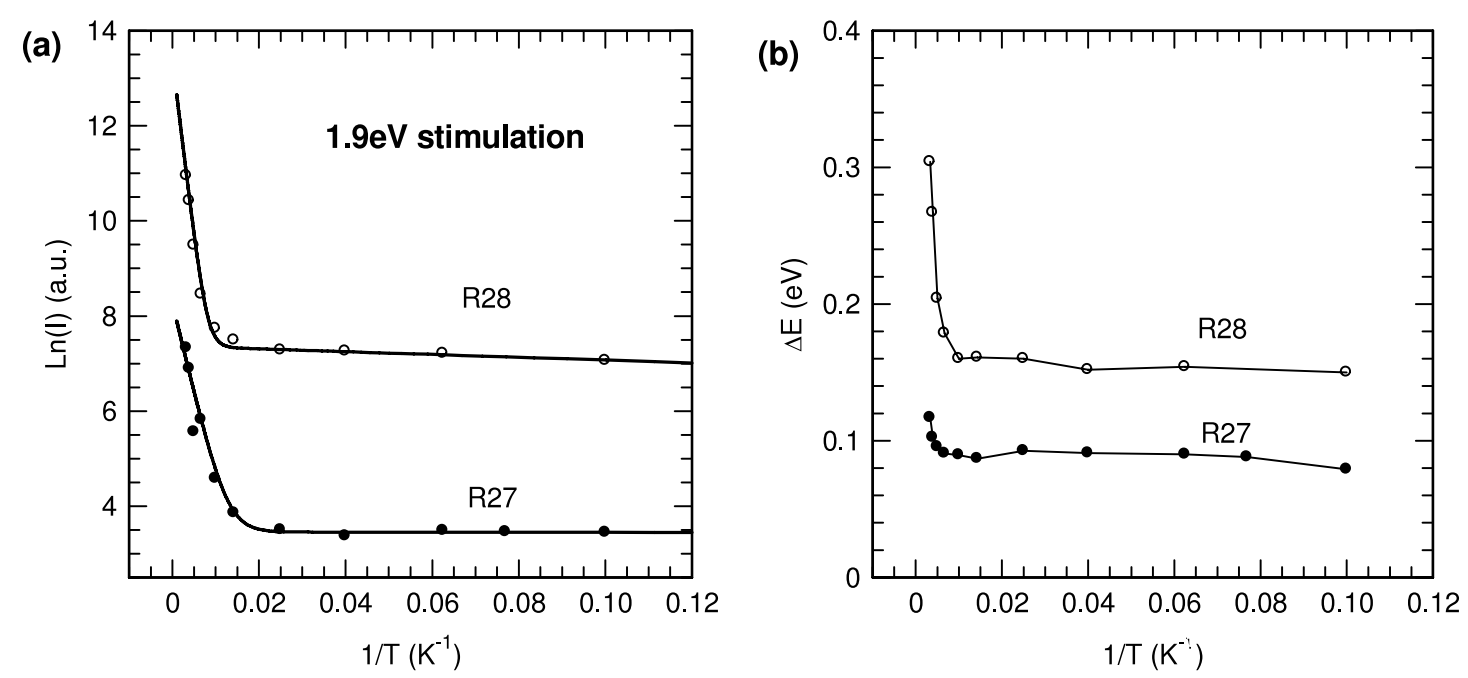

Figure 5. Temperature dependence of the non-resonant continuum transitions shown in figure 2 (a) for the intensity of the transition under $1.9 \mathrm{eV}$ stimulation and (b) width of the exponential tail, $\Delta E$.

(1988) and Poolton et al (1995a, 1995b) considered that $E_{\mathrm{d}}$ would be either $\sim 2.5 \mathrm{eV}$ or $2 \mathrm{eV}$, respectively. For the purposes of this work, the exact value is not of relevance, except to note that from all previous evidence, it is certain to be $>2 \mathrm{eV}$ : potentially, it is the band-tails themselves as assessed in this work that probably causes difficulty in obtaining an accurate value for $E_{\mathrm{d}}$. As such, a direct defect ionization processes that accesses the high mobility conduction band (equation (3)) cannot therefore explain the rising non-resonant OSL response with energy shown in figure 2 . Previously, we argued that such continua are more typical of that expected for a continuum of band-tail states and as such, if the luminescence efficiency directly follows their density of states, the energy dependence would be expected to be of the form:

$$
\operatorname{OSL}(E) \propto \exp \left(\frac{E-E_{\mathrm{d}}}{\Delta E}\right) .
$$

Here, $\Delta E$ is the width of the band-tail states accessible from the ground state of the OSL defect. (Note that $\Delta E$ is not necessarily the same as the width of the Urbach tail, as the ground state could, in principle, lie embedded within the Urbach tail-in which case, there would be further states below the OSL ground state that are inaccessible.) The rising continua in figure 2 are shown to fit this equation well and the thermal dependence of both the OSL intensity (at $1.9 \mathrm{eV}$ stimulation energy) and of $\Delta E$ are given in figure 5. ${ }^{4}$ The intensity of the signals follow very closely the temperature dependence of the resonances, and the Arrhenius plots would again suggest both a thermally active and a non-thermally activated process to the OSL. For R27 and R28, the Arrhenius plots yield activation energies of $0.033 \mathrm{eV}$ and $0.065 \mathrm{eV}$ respectively (and both yielding $0.000 \mathrm{eV}$ at temperatures below

\footnotetext{
4 Whilst $\Delta E$ is an unambiguous constant that can be obtained from any individual fit, the value of $E_{\mathrm{d}}$ is arbitrary if the proportionality constant can be varied. However, it is not expected that $E_{\mathrm{d}}$ would change with temperature, so equation (4) can be used to determine the temperature dependence of both $\Delta E$ and the OSL intensity at any excitation energy. An arbitrary free-fit value for $E_{\mathrm{d}}$ of $\sim 2.06 \mathrm{eV}$ was, in fact, obtained for the curves at all temperatures, giving confidence to the fit values for the proportionality constant.
}

$50 \mathrm{~K})$. As seen from figure 5(b), between 300 and $100 \mathrm{~K}$, the width of the accessible trapping states changes from 0.30 to $0.16 \mathrm{eV}$ for R28, and between 0.12 and $0.09 \mathrm{eV}$ for R27: below $100 \mathrm{~K}$, the widths are invariant with temperature.

The temperature dependence of the OSL intensity of the continua can be explained in terms of excitation from the OSL defect ground state, directly to the band-tail states, from where thermally activated hopping allows transport to the recombination sites; at very low temperatures, however, where this hopping mechanism is essentially frozen out, luminescence can still proceed via tail-to-tail (or tail-torecombination site) tunnelling processes, which would be non-thermal. Whilst we believe this to be the most likely explanation, an alternative does exist-and therefore needs to be considered. In the original work of Urbach (1953) concerning tail states probed by near-band-edge optical absorption in silver halides, the parameter $\Delta E$ (which in this case constitutes the full Urbach tails) was shown to be thermally dependent, following a Boltzmann distribution law. However, in a number of materials, this Boltzmann distribution breaks down at low temperatures, and a more complex expression is needed. It has been argued that the model needs to take into account the zero-point energy of the lattice (e.g. see Mahr 1962, Akimoto et al 1996): in so doing the temperature dependence of the OSL at any given (fixed) excitation energy would be expected to follow the form

$$
\operatorname{OSL}(T) \propto \exp \left[\frac{2 \sigma_{0}}{E_{\mathrm{ph}}} \tanh \left(\frac{E_{\mathrm{ph}}}{2 k T}\right)\right]
$$

where $k$ is the Boltzmann constant, $\sigma_{0}$ a constant characteristic of the material and $E_{\mathrm{ph}}$ the average energy of the phonons that contribute to the Urbach tail. The hyperbolic function enables the asymptotic approach to the zero-point energy as the sample temperature is reduced. As shown in figure 6 (for the case of $\mathrm{NaAlSi}_{3} \mathrm{O}_{8}$ sample R27) such a function can very well account for the temperature dependence of both the resonant and non-resonant OSL transition over the whole temperature range $10-300 \mathrm{~K}$. However, the reason that this 


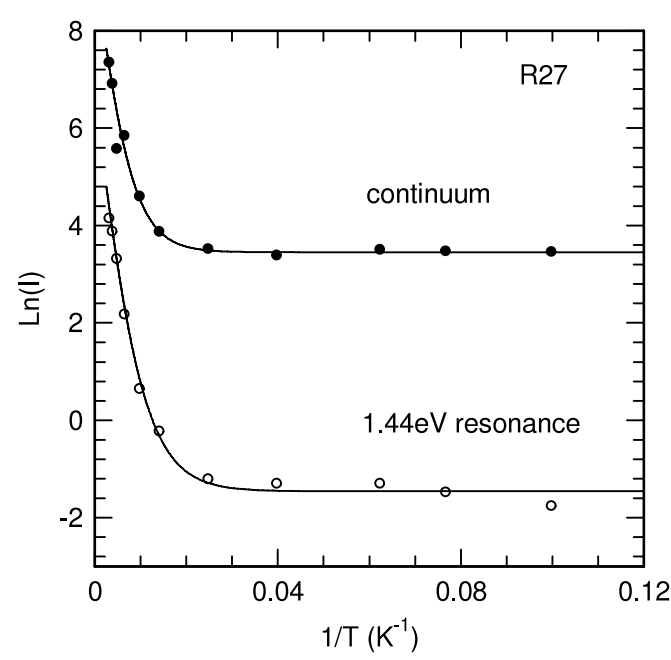

Figure 6. As demonstrated for the $\mathrm{NaAlSi}_{3} \mathrm{O}_{8}$ sample, the temperature dependence of the integrated $1.44 \mathrm{eV}$ resonance, and intensity of the continuum OSL excitation spectra (for sample irradiation at $300 \mathrm{~K}$ ) can be accounted for over the full range $10-300 \mathrm{~K}$ (solid lines) if equation (5) is used, which accounts for zero-point energy fluctuations. The form is similar to the transition between thermal and non-thermal processes shown in figure 3 .

mechanism is unlikely resides in the values of $E_{\mathrm{ph}}$ obtained from the fitting (20 meV and $16 \mathrm{meV}$ for the continuum and $1.44 \mathrm{eV}$ resonant transitions, respectively). These energies are far too small to account for the main lattice phonons. As summarized by Salje (1993), weak vibrational features in the 10-25 meV (80-200 $\mathrm{cm}^{-1}$ ) energy range are found for (NaK) $\mathrm{AlSi}_{3} \mathrm{O}_{8}$ but, as with most other silicate materials, the dominant phonons mainly occur within the range $35-60 \mathrm{meV}$ (300-500 $\mathrm{cm}^{-1}$ ); these latter values correspond directly with the thermal activation energies determined in the preceding section.

\subsection{Optically and thermally probing band-tail states populated at low temperature}

Hitherto, the results described in this paper concern material that has been irradiated at room temperature, and for which OSL measurements have been undertaken at lower temperatures; this ensures that the origin of the trapped electrons are the OSL defects themselves, and the means of charge transfer to the recombination centres (e.g. via the bandtail states) can be probed. However, if such a continuum of band-tail states actually exists, then it would be anticipated that if the material is irradiated at $10 \mathrm{~K}$, charge carriers (both electrons and holes) could become trapped within the continuum of states that lie below the conduction band, and above the valence band. As such, both the energy-resolved OSL stimulation spectra and TL should show broad continua reflecting the density of band-tail states. In the case of the OSL stimulation, figure 7(a) shows precisely this effect for both materials studied, where $10 \mathrm{~K}$ is both the irradiation and measurement temperature. The OSL is totally different from that observed with room temperature irradiation and readout, and consists simply of an intense continuum, rising as a function of the excitation energy. It is interpreted in terms of excitation of electrons trapped in the band-tail into the conduction band (well clear of the band edge at these energies), from where they are free to recombine at hole trapping centres. In such a scenario, OSL would be expected to proceed under general order kinetics, as given in equation (1). As shown in figure 7(b) for R27, this is indeed found to be the case, where the parameters $\alpha$ and $\beta$ are fitted as 0.02 and 1.0 respectively. (Here, the excitation energy was chosen as $1.44 \mathrm{eV}$-i.e. close to the IRSL resonance-to emphasize the difference in kinetics between IRSL excitation from the band-tails themselves, and from charge located at the deep trapping centres). If the bandtails are taken to be exponential, then to a first approximation, the energy-dependent OSL stimulation spectra should follow the form of equation (6):

$$
\operatorname{OSL}(E) \propto \exp \left(\frac{E}{E_{\mathrm{U}}}\right)
$$

where in this case, $E_{\mathrm{U}}$ is the true (Urbach) band-tail width. This is only an approximation and is limited in energy range, since clearly charge in the shallowest tails would quickly disperse due to tunnelling transport (and subsequent
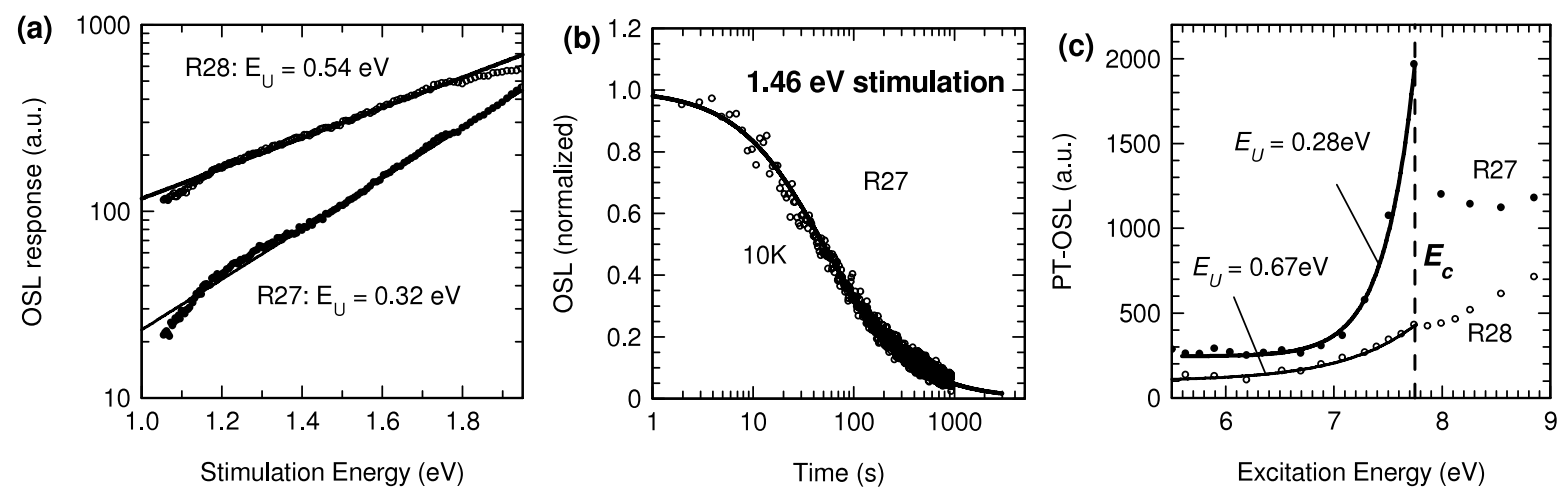

Figure 7. Characteristics of OSL in the feldspar samples following irradiation a $10 \mathrm{~K}$. (a) Stimulation spectrum following $\mathrm{x}$-irradiation for both the $\mathrm{Na}$ and $\mathrm{K}$ sample. Unlike the structured spectra of figure 2, the spectra are simply continua, fitted to exponentials (full curves) that follow equation (5): the Urbach energies, $E_{\mathrm{U}}$, obtained in the fits are shown. (b) The OSL decay in the $\mathrm{NaAlSi}_{3} \mathrm{O}_{8}$ is fitted to a general order kinetics equation (full curve). (c) Photo-transferred OSL in the near band edge excitation regime (OSL read-out using $532 \mathrm{~nm}$ laser). The sub-high-mobility edge Urbach tails are fitted using the $E_{\mathrm{U}}$ energies indicated (solid lines) for both the Na and $\mathrm{K}$ feldspar samples. The values are nearly identical to those shown in (a), indicating they both are different measurements of the same phenomenon. 

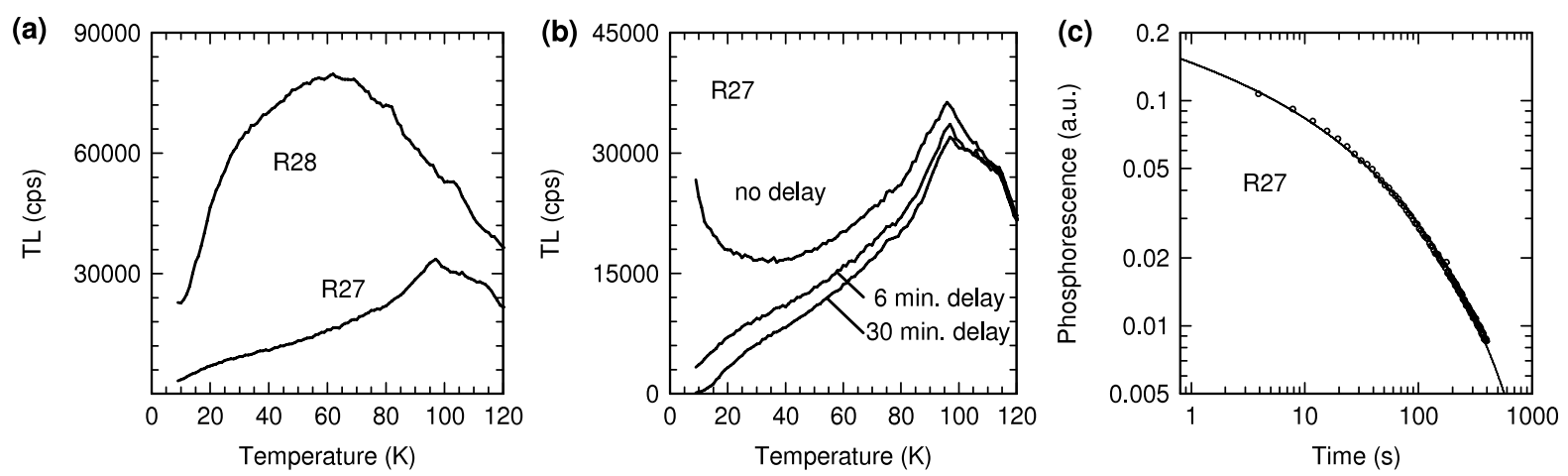

Figure 8. (a) Thermoluminescence from K- (R28) and Na- (R27) feldspars, after x-irradiation at $10 \mathrm{~K}$, demonstrating the TL continuum deriving from band-tail states. (b) The shallowest band-tail states become depleted with time, due to tunnelling processes-shown here for R27. (c) This tunnelling process is radiative, and can be fitted to the tunnelling equation (7) (solid line), shown here for R27.

recombination) thus would not remain populated, even at $10 \mathrm{~K}$. Furthermore, the form needs to take into consideration the energy dependence of the density of states (DOS) of the conduction band proper, which is unknown. However if it does approximate to a parabolic, then within the energy range studied $(1-2 \mathrm{eV})$, the DOS in the true conduction band is unlikely to vary significantly. With these approximations in mind, a substantial portion of the stimulation curves do indeed follow equation (6), with the tail widths $E_{\mathrm{U}}$ being determined as $0.32 \mathrm{eV}$ and $0.54 \mathrm{eV}$ in the $\mathrm{NaAlSi}_{3} \mathrm{O}_{8}$ and $\mathrm{KAlSi}_{3} \mathrm{O}_{8}$ samples, respectively. Reassuringly, these figures are entirely consistent with data we previously measured (but not analysed as such) using synchrotron radiation in the near band edge region of the materials (Poolton et al 2006): at $10 \mathrm{~K}$, synchrotron photons were used to populate the band-tails, and then $532 \mathrm{~nm}$ laser light $(2.33 \mathrm{eV})$ is used in a subsequent OSL read-out (Photo-transferred OSL, PTOSL). The pertinent results are shown in figure 7 (c); analysing this data in the context of the present work, it is clear that up to the band edge $E_{\mathrm{c}}$, the signals can be fitted to the relevant equation

$$
\operatorname{PTOSL}(E) \propto \exp \left(\frac{E-E_{\mathrm{c}}}{E_{\mathrm{U}}}\right) .
$$

The values derived for the Urbach tails in R27 and R28 from the synchrotron experiments are $0.28 \mathrm{eV}$ and $0.67 \mathrm{eV}$ respectively-essentially the same values as obtained from the low temperature OSL stimulation spectra measured here ( $0.32 \mathrm{eV}$ and $0.54 \mathrm{eV}$ respectively). Interestingly, these values of $E_{\mathrm{U}}$ compare with the values of $\Delta E$ obtained at $300 \mathrm{~K}$ of $0.12 \mathrm{eV}$ and $0.31 \mathrm{eV}$ respectively for the two materials, indicating that large portions of the tails are being accessed via excitation for the OSL ground state at room temperature.

In corollary with the continua observed in the OSL stimulation spectra, the thermoluminescence curves of both samples (figure 8(a)) also display strong, but featureless emission within the temperature range $10-80 \mathrm{~K}$, which we interpret as thermal excitation from charge trapped in the bandtail continuum. Above $80 \mathrm{~K}$, more discrete trapping sites can be observed superimposed on the continuum. Both samples display strong phosphorescence following the cessation of $\mathrm{x}$ irradiation at $10 \mathrm{~K}$ and, if the TL read-out is delayed, the loss of the trapped charge from the shallowest band-tails can be monitored, as shown if figure 8(b): figure 8(c) indicates this is a direct tunnelling processes, since the time-dependent phosphorescence can be very well fitted to the tunnelling luminescence decay equation given by Delbecq et al (1974):

$$
I_{\mathrm{phos}}(t)=C \frac{1}{t_{0}} \ln \left[1+\frac{t_{0}}{t}\right]
$$

where $C$ is a proportionality constant and $t_{0}$ is the irradiation time (needed since the initial irradiation is not an instant pulse, so that some pairs that are created early on during the irradiation are already starting to recombine before the irradiation ceases). In the free fit, $t_{0}=211$, close to the actual value, $240 \mathrm{~s}$.

Because there are no peaks that can be fitted to obtain the trapping parameters of individual band-tail states, it is difficult to correlate the depth of a particular state to the temperature at which it induces TL. However, Strickertsson (1985) has previously correlated TL peak position with thermal trap depth in $\mathrm{K}$ feldspars for measurements extending above room temperature involving discrete defect levels, and these lie on a continuous curve that would extend to $0 \mathrm{eV}$ at $0 \mathrm{~K}$ (see figure 9(a)). This can only be regarded as a crude analogy, but within this framework, the thermal trap depths sampled in the range $0-100 \mathrm{~K}$ would be expected to lie within the energy range $0-0.3 \mathrm{eV}$, i.e. in the same range as the bandtail widths determined here using OSL excitation spectroscopy. Figure 9(b) shows the theoretical TL spectra that would ensue from individual tail states that give rise to the continuum, for a range of values $0.05-0.4 \mathrm{eV}$ (assuming first order kinetics, an escape frequency factor of $10^{13} \mathrm{~s}^{-1}$, and ramp rate of $10 \mathrm{~K} \mathrm{~min}^{-1}$, as used in this experiment). We have found such TL continua in all feldspar samples measured so far, without exception; the only other previous low temperature work we are aware of is by Visocekas et al (1998), who observed similar spectral features and also tentatively attributed them to the presence of band-tail states. We also note that similar continua have been reported in other disordered materials (e.g. Kadashchuk et al 1998).

The final and most definitive proof that charge can be transferred from the OSL centres to the band-tail states is 

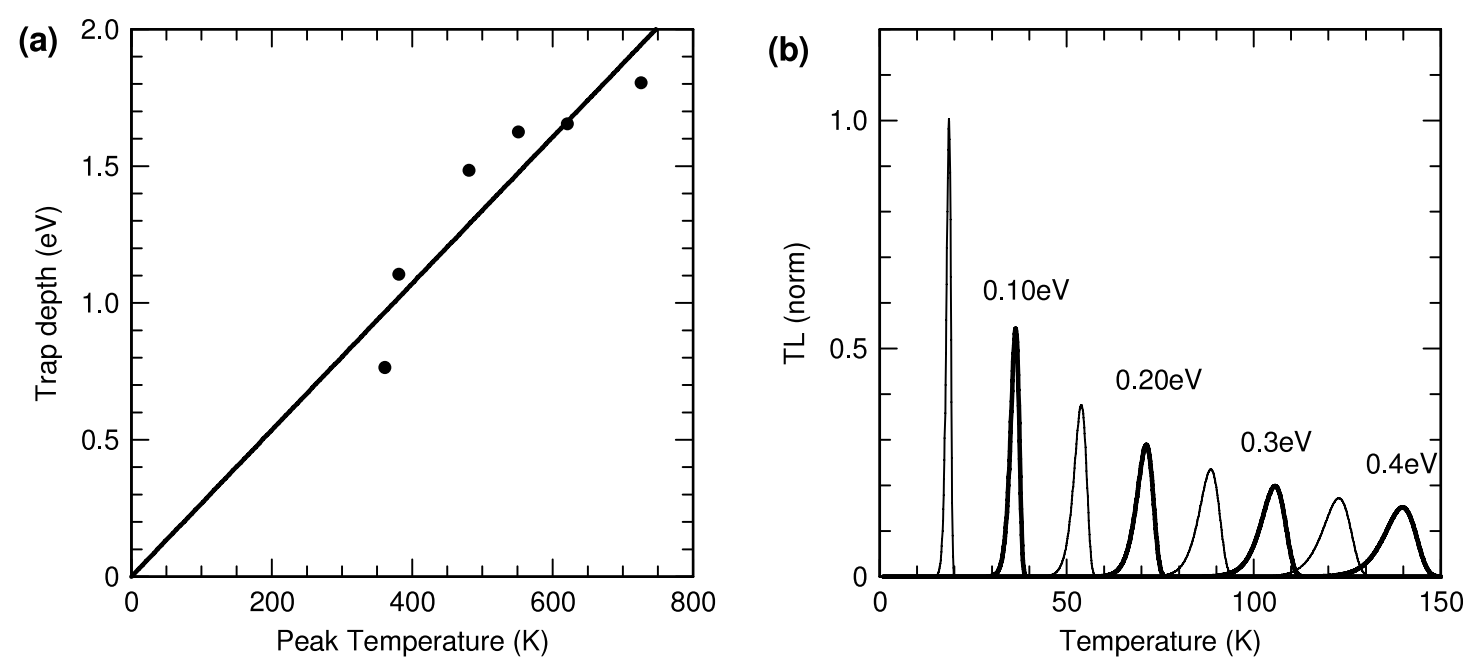

Figure 9. (a) Plot of the thermal trap depth versus TL peak position for high temperature measurements in K feldspar, extrapolated to $0 \mathrm{~K}$; data from Strickertsson (1985); this provides a rough energy calibration for the data given in figure 8(a). (b) Hypothetical TL peaks (50 meV spacing) calculated for charge released from individual trapping sites within the band-tail continuum, given first order kinetics, a ramp rate of $10 \mathrm{~K} \mathrm{~min}^{-1}$, and escape frequency factor $s=10^{13} \mathrm{~s}^{-1}$.

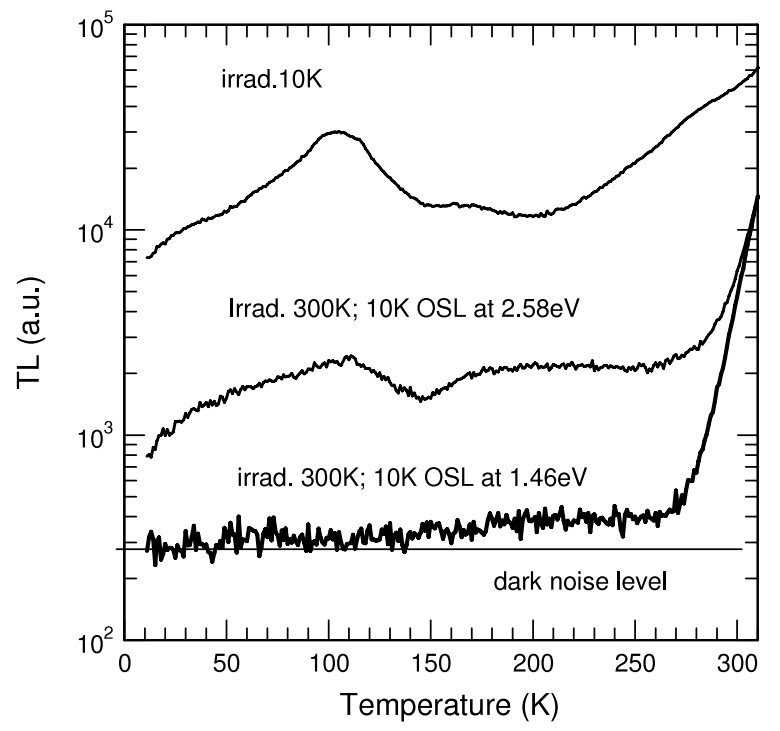

Figure 10. Comparison of the photo-transferred TL from OSL-active sites populated at $300 \mathrm{~K}$ (but transferred at $10 \mathrm{~K}$ ) with the TL trap spectrum of material $\mathrm{x}$-irradiated at $10 \mathrm{~K}$, for the $\mathrm{Na}$ feldspar sample R27. The diagram demonstrates that $480 \mathrm{~nm}$ photons transfers charge via the high mobility conduction band, as the full energy distribution of band-tail states are populated, whereas $850 \mathrm{~nm}$ photons accesses only deeper-lying band-tail states, as only the higher temperature TL traps are populated. The sharp rise in the TL above $260 \mathrm{~K}$ results from deep-level traps already populated during $300 \mathrm{~K}$ irradiation.

via photo-transferred TL measurements. If the samples are irradiated at $300 \mathrm{~K}$, the OSL centres will become populated, but not the band-tails. If the sample is now reduced in temperature to $10 \mathrm{~K}$ and illuminated with light, the final destination of much of that charge will be frozen, but the location can be determined by subsequently undertaking TL between 10 and $300 \mathrm{~K}$. The results of this experiment are shown in figure 10 for the case of R27; here it can clearly be seen that illumination at $480 \mathrm{~nm}(2.58 \mathrm{eV})$ populates the full spectrum of trapping states from $10 \mathrm{~K}(\sim 0.02 \mathrm{eV})$ upwards (which would otherwise have been empty), strongly indicating the ionization of the OSL centres via electron transfer through the high mobility conduction band: the spectrum is indistinguishable from that of the sample having been $\mathrm{x}$-irradiated at low temperature. In contrast, illumination in the IR at $850 \mathrm{~nm}(1.46 \mathrm{eV})$ only populates TL trapping sites that are depopulated at temperatures greater than $140 \mathrm{~K}$ ( $\sim 0.4 \mathrm{eV}$ according to figure $9(\mathrm{a})$ ). This value is important: it implies that if the optical trap depth $E_{\mathrm{d}}$ of the OSL centre does lie in the suggested range $2-2.5 \mathrm{eV}$ as discussed in section 3.2, the defect's excited state does lie firmly embedded within the thermally accessible band-tail continuum, implying the timestability of charge located at the defect would be considerably shorter than that expected if the band-tail states did not exist.

\section{Conclusions}

The unique set of low temperature experiments undertaken in this work shows conclusively that the previously postulated continuum of band-tail states of naturally occurring aluminosilicate minerals (feldspars) both exist, and play a crucial role in the luminescence and charge transport properties of the materials. The extent of the tails, $E_{\mathrm{U}}$, has been directly determined to be around $0.4 \mathrm{eV}$ in the typical $\mathrm{NaAlSi}_{3} \mathrm{O}_{8}$ and $\mathrm{KAlSi}_{3} \mathrm{O}_{8}$ materials studied; as such, this means that the excited state of the ubiquitous IRSL dosimetry trapping state lies deeply embedded in this continuum, which allows chargecarrier transport through the lattice via band-tail tunnelling or band-tail hopping when charge is stimulated from the ground, to excited state.

\section{Acknowledgments}

We thank Ole Johnsen of Copenhagen University Geological Museum for the loan of the samples used. The work is 
funded by EPSRC (UK: grant EP/F065272/1), the Technology

Foundation STW (The Netherlands: grant 10502) and Delft University of Technology (TUD Grant TTQ700).

\section{References}

Aitken M 1998 An Introduction to Optical Dating (Oxford: Oxford University Press)

Akimoto R, Kobayashi M and Suzuki T 1996 The Urbach tail of absorption and photoluminescence spectra in EuSe J. Phys.: Condens. Matter 8 105-10

Bailiff I K and Barnett S M 1994 Characteristics of infrared-stimulated luminescence from a feldspar at low temperatures Radiat. Meas. 23 541-5

Bailiff I K and Poolton N R J 1991 Studies of charge transfer mechanisms in feldspars Nucl. Tracks Radiat. Meas. 18 111-8

Barnett S M and Bailiff I K 1997 Infrared stimulation spectra of sediments containing feldspar Radiat. Meas. 27 237-42

Baril M R and Huntley D J 2003 Optical excitation spectra of trapped electrons in irradiated feldspars J. Phys.: Condens. Matter 15 8011-27

Bøtter-Jensen L, McKeever S W S and Wintle A G 2003 Optically Stimulated Luminescence Dosimetry (Amsterdam: Elsevier)

Clark R J and Sanderson D C W 1994 Photostimulated luminescence excitation spectroscopy of feldspars and micas Radiat. Meas. 23 641-6

Delbecq C J, Toyozawa Y and Yuster P H 1974 Tunnelling recombination of trapped electrons and holes in $\mathrm{KCl}: \mathrm{AgCl}$ and KCl:TlCl Phys. Rev. B $94497-505$

Duller G A T and Wintle A G 1991 On infrared stimulated luminescence at elevated temperatures $\mathrm{Nucl}$. Tracks Radiat. Meas. 18 379-84

Huntley D J 2006 An explanation of the power-law decay of luminescence J. Phys.: Condens. Matter 18 1359-65

Huntley D J and Lamothe M 2001 Ubiquity of anomalous fading in $\mathrm{K}$-feldspars and the measurement and correction for it in optical dating Can. J. Earth Sci. 38 1093-106

Hütt G, Jaek I and Tchonka J 1988 Optical dating: K-feldspar optical response stimulation spectra Quat. Sci. Rev. 7 381-5

Itoh C, Tanimura K, Itoh N and Itoh M 1989 Threshold energy for photogeneration of self-trapped excitons in $\mathrm{SiO}_{2}$ Phys. Rev. B 39 11183-6

Kadashchuk A, Ostapenko N, Zaika V and Nešpùrek S 1998 Low temperature thermoluminescence in poly(methyl-phenylsilylene) Chem. Phys. 234 285-96

Kars R H, Wallinga J and Cohen K M 2008 A new approach towards anomalous fading correction for feldspar IRSL dating - tests on samples in field saturation Radiat. Meas. 43 786-90
Mahr H 1962 Ultraviolet absorption of KI diluted in $\mathrm{KCl}$ crystals Phys. Rev. 125 1510-6

Malins A E R, Poolton N R J, Quinn F M, Johnsen O and Denby P M 2004 Luminescence excitation characteristics of $\mathrm{Ca}, \mathrm{Na}$ and $\mathrm{K}$ aluminosilicates (feldspars) in the stimulation range $5-40 \mathrm{eV}$ : determination of the band-gap energies J. Phys. D: Appl. Phys. 37 1439-50

McKeever S W S, Banerjee D, Blair M, Clifford S M, Clowdsly M S, Kim S S, Lamothe M, Lepper K, Leuschen M, McKeever K J, Prather M, Rowland A, Reust D, Sears D W G and Wilson J W 2003 Concepts and approaches to in situ luminescence dating of Martian sediments Radiat. Meas. 37 527-34

Megaw H 1974 The architecture of feldspars The Feldspars ed W S MacKenzie and J Zussman (Manchester: Manchester University Press) pp 1-24

Poolton N R J, Bøtter-Jensen L and Johnsen O 1995a Influence of donor electron energies of the chemical composition of $\mathrm{K}, \mathrm{Na}$ and Ca aluminosilicates J. Phys.: Condens. Matter 7 4751-62

Poolton N R J, Bøtter-Jensen L and Johnsen O 1995b Thermo-optical properties of optically stimulated luminescence in feldspars Radiat. Meas. 24 531-4

Poolton N R J, Ozanyan K B, Wallinga J, Murray A S and

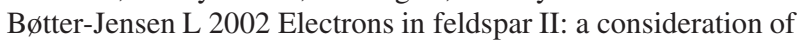
the influence of conduction band-tail states on luminescence processes Phys. Chem. Miner. 29 217-25

Poolton N R J, Mauz B, Lang A, Jain M and Mailins A E R 2006 Optical excitation processes in the near band-edge region of $\mathrm{KAlSi}_{3} \mathrm{O}_{8}$ and $\mathrm{NaAlSi}_{3} \mathrm{O}_{8}$ feldspar Radiat. Meas. 41 542-8

Poolton N R J, Bos A J J, Wallinga J, de Haas J T M, Dorenbos P, de Vries L, Kars R H, Jones G O and Drozdowski W 2009 Non-resonant $\mathrm{X}$-ray pump/laser probe spectroscopy as a method for assessing charge competition, trapping and luminescence efficiency in wide band-gap materials, submitted

Rieser U, Hütt G, Krbetschek M R and Stoltz W 1997 Feldspar IRSL emission spectra at high and low temperatures Radiat. Meas. $27273-8$

Salje E K H 1993 Phase transitions and vibrational spectroscopy in feldspars Feldspars and their Reactions ed I Parsons (Dordrecht: Kluwer Academic) pp 103-60

Strickertsson K 1985 The thermoluminescence of potassium feldspars-glow curve characteristics and initial rise measurements $\mathrm{Nucl}$. Tracks 10 613-7

Urbach F 1953 The long-wavelength edge of photographic sensitivity and of the electronic absorption of solids Phys. Rev $\mathbf{9 2} 1324$

Visocekas R, Tale V, Zink A and Tale I 1998 Trap spectroscopy and tunnelling luminescence in feldspars Radiat. Meas. 29 427-34

Wintle A G 1973 Anomalous fading of thermoluminescence in mineral samples Nature 245 143-4 